\title{
Diagnosis of Obscure Gastrointestinal Bleeding
}

\author{
Satoshi Tanabe \\ Division of Therapeutic Endoscopy, Department of Advanced Medicine, Research and Development Center for New Medical Frontiers, \\ Kitasato University School of Medicine, Sagamihara, Japan
}

\begin{abstract}
Obscure gastrointestinal bleeding (OGIB) is defined as gastrointestinal bleeding from a source that cannot be identified on upper or lower gastrointestinal endoscopy. OGIB is considered an important indication for capsule endoscopy (CE). CE is particularly useful for the detection of vascular and small ulcerative lesions, conditions frequently associated with OGIB, particularly in Western countries. On the other hand, balloon enteroscopy (BE) can facilitate the diagnosis of lesions presenting with minimal changes of the mucosal surface, such as submucosal tumors, and can be used not only for diagnosis, but also for treatment, including endoscopic hemostasis. In other words, the complementary use of both $\mathrm{CE}$ and BE enables OGIB to be more efficiently diagnosed and treated. However, rebleeding can occur even in patients with negative results of $\mathrm{CE}$, and such patients require repeat $\mathrm{CE}$ or $\mathrm{BE}$. It is essential to effectively use both $\mathrm{CE}$ and $\mathrm{BE}$ based on a thorough understanding of the strong points and weak points of these procedures. Further advances and developments in virtual endoscopy incorporating computed tomography and magnetic resonance imaging are expected in the future. Clin Endosc 2016;49:539-541
\end{abstract}

Key Words: Obscure gastrointestinal bleeding; Capsule endoscopy; Balloon enteroscopy

\section{INTRODUCTION}

Obscure gastrointestinal bleeding (OGIB) is defined as recurrent or persistent gastrointestinal bleeding from a source that cannot be identified using upper or lower endoscopy, and is associated with a so-called "gastrointestinal disease of unknown cause." OGIB occurs in approximately $5 \%$ of all patients with gastrointestinal bleeding. ${ }^{2}$ In $41 \%$ to $75 \%$ of patients with OGIB, further evaluation can confirm the lesions that cause bleeding in the small intestine. ${ }^{3}$

The anatomic features of the small intestine rendered conventional endoscopy difficult and often precluded evaluation of the entire organ. Capsule endoscopy (CE) was developed in

Received: December 3, 2015 Revised: January 20, 2016

Accepted: January 20, 2016

Correspondence: Satoshi Tanabe

Division of Therapeutic Endoscopy, Department of Advanced Medicine, Research and Development Center for New Medical Frontiers, Kitasato University School of Medicine, 1-15-1 Kitasato, Minami, Sagamihara 252-0374, Japan Tel: +81-42-778-8111, Fax: +81-42-778-8390, E-mail: s-tanabe@kitasato-u.ac.jp

(c) This is an Open Access article distributed under the terms of the Creative Commons Attribution Non-Commercial License (http://creativecommons.org/ licenses/by-nc/3.0) which permits unrestricted non-commercial use, distribution, and reproduction in any medium, provided the original work is properly cited.
$2000,{ }^{4}$ followed by double-balloon enteroscopy (DBE) in $2001^{5}$ and single-balloon enteroscopy (SBE) in 2007. These new endoscopic techniques greatly impacted the diagnosis and treatment of small-bowel disease and enabled the endoscopic diagnosis and treatment of lesions located in the deep small intestine. In this review, we evaluated the diagnosis of OGIB.

\section{DIAGNOSTIC ALGORITHM AND MANAGEMENT OF OGIB}

A diagnostic algorithm for OGIB was proposed by the International Congress of Capsule Endoscopy. With the exception of angiography based on interventional radiography, CE has been designated as the diagnostic technique of choice for patients with massive bleeding. ${ }^{3,7}$ Ohmiya et al. ${ }^{8}$ reported that in patients with both overt and occult OGIB, a cross-sectional imaging technique, contrast-enhanced helical computed tomography (CT), should initially be performed to evaluate small-bowel disease in the absence of any contraindications such as renal failure or a history of allergy to contrast agents. In case abnormalities are detected on abdominal CT, 
deep enteroscopy such as DBE, SBE, and spiral enteroscopy, should be performed via the oral or anal approach depending on the suspected lesion site.

\section{CAPSULE ENDOSCOPY}

$\mathrm{CE}$ is associated with minimal physical burden on patients and involves a simple procedure, with the exception of capsule retention. It is therefore considered an appropriate modality for screening examinations. In fact, Liao et al. ${ }^{9}$ reported that $\mathrm{CE}$ is used as the technique of choice for the evaluation of OGIB in $66 \%$ of hospitals owing to its convenience and noninvasiveness. The diagnostic accuracy of CE for OGIB is $41 \%$ to $80 \%$ as compared with that of DBE, which is considered the gold standard for visualization of the small bowel. ${ }^{3} \mathrm{CE}$ enables easy detection of vascular lesions as well as small ulcerative lesions. Because CE images are recorded through a transparent dome, often when the capsule is in contact with the intestinal wall, magnified images of the lesions are obtained. However, CE-assisted diagnosis of lesions with an intact mucosal surface is difficult, such as submucosal tumors. Such bulky lesions are easily diagnosed through imaging studies, performed using balloon enteroscopy (BE) or CT. The use of a combination of various examination techniques can reduce the non-detection rate.

\section{BALLOON ENTEROSCOPY}

$\mathrm{BE}$ is slightly more invasive than $\mathrm{CE}$ is, but enables access to all parts of the small intestine; moreover, it allows one to perform procedures generally similar to procedures that can be performed using conventional endoscopy. Two types of balloon enteroscopes are currently commercially available: DBE and SBE. Both these enteroscopes require different insertion techniques depending on the presence or absence of a balloon at the enteroscope tip. However, the principle of insertion is the same: the enteroscope is inserted while pleating the small intestine onto a sliding tube or overtube. May et al. ${ }^{10}$ conducted a randomized controlled study to compare the push-and-pull enteroscopy performed using a DBE (Fujifilm Corp., Tokyo, Japan) with that performed using a SBE. The same enteroscopic system was used to perform both the procedures; however, a balloon was not present at the tip of the SBE. The success rate of complete small-bowel examination was significantly higher when DBE (66\%) was used than when SBE (22\%) was used ( $p<0.0001)$; however, the rate of positive findings did not differ between DBE (52\%) and SBE (42\%; not significant [NS]). Domagk et al. ${ }^{11}$ performed a randomized trial to compare DBE (EN450-P5/T5; Fujifilm Corp.) with SBE (SIF-Q180; Olympus Co. Ltd., Tokyo, Japan) and found no significant difference in the success rate of complete small-bowel examination (DBE, $18 \%$ vs. SBE, $11 \%$; NS) or the rate of positive findings (DBE, $43 \%$ vs. SBE, $37 \%$; NS). A meta-analysis of 11 rather large studies of the overall diagnostic rates showed that the diagnostic accuracy of $\mathrm{CE}$ $(60 \%)$ was similar to that of $\mathrm{BE}(57 \%){ }^{12}$

\section{NEGATIVE RESULTS OF CE AND ACUTE OVERT OGIB}

CE is a useful modality for the diagnosis of OGIB. However, bleeding can recur even after $\mathrm{CE}$ examinations yield negative results. Repeat $\mathrm{CE}$ is indicated in the case of patients who show rebleeding. $\mathrm{BE}$ is also useful in the case of patients who show negative results on CE examination. ${ }^{13} \mathrm{~A}$ recent study showed that emergency DBE combined with real-time viewing with $\mathrm{CE}$ is useful in the case of patients with acute overt OGIB. ${ }^{14}$ This technique facilitated the detection of recurrent vascular lesions, such as Dieulafoy's lesions, which are easily overlooked on elective DBE. In any case, both CE and BE are essential and play complementary roles in the diagnosis and treatment of OGIB.

\section{CONCLUSIONS}

We presented the method for the diagnosis of OGIB along with recent findings. Examination of the entire small intestine by using conventional endoscopy was difficult. However, the development of, and progress in, $\mathrm{CE}$ and $\mathrm{BE}$ have led to the establishment of effective techniques for enteroscopic examination and resulted in many benefits for physicians as well as patients. However, the patient's background characteristics and general condition at the time of diagnosis should certainly be considered, without relying solely on the results of endoscopic examinations. Both CE and BE should be effectively used after the advantages and disadvantages of each technique have been understood. Further progress and developments in virtual endoscopy, such as the incorporation of CT and magnetic resonance imaging, are expected in the future.

Conflicts of Interest

The author has no financial conflicts of interest. 


\section{REFERENCES}

1. Zuckerman GR, Prakash C, Askin MP, Lewis BS. AGA technical review on the evaluation and management of occult and obscure gastrointestinal bleeding. Gastroenterology 2000;118:201-221.

2. Szold A, Katz LB, Lewis BS. Surgical approach to occult gastrointestinal bleeding. Am J Surg 1992;163:90-92; discussion 92-93.

3. Raju GS, Gerson L, Das A, Lewis B; American Gastroenterological Association. American Gastroenterological Association (AGA) Institute technical review on obscure gastrointestinal bleeding. Gastroenterology 2007;133:1697-1717.

4. Iddan G, Meron G, Glukhovsky A, Swain P. Wireless capsule endoscopy. Nature 2000;405:417

5. Yamamoto H, Sekine $Y$, Sato $Y$, et al. Total enteroscopy with a nonsurgical steerable double-balloon method. Gastrointest Endosc 2001;53:216220

6. Tsujikawa T, Saitoh Y, Andoh A, et al. Novel single-balloon enteroscopy for diagnosis and treatment of the small intestine: preliminary experiences. Endoscopy 2008;40:11-15.

7. Raju GS, Gerson L, Das A, Lewis B; American Gastroenterological Association. American Gastroenterological Association (AGA) Institute medical position statement on obscure gastrointestinal bleeding. Gas- troenterology 2007;133:1694-1696.

8. Ohmiya N, Nakagawa Y, Nagasaka M, et al. Obscure gastrointestinal bleeding: diagnosis and treatment. Dig Endosc 2015;27:285-294.

9. Liao Z, Gao R, Xu C, Li ZS. Indications and detection, completion, and retention rates of small-bowel capsule endoscopy: a systematic review. Gastrointest Endosc 2010;71:280-286.

10. May A, Färber M, Aschmoneit I, et al. Prospective multicenter trial comparing push-and-pull enteroscopy with the single- and double-balloon techniques in patients with small-bowel disorders. Am J Gastroenterol 2010;105:575-581.

11. Domagk D, Mensink P, Aktas H, et al. Single- vs. double-balloon enteroscopy in small-bowel diagnostics: a randomized multicenter trial. Endoscopy 2011;43:472-476.

12. Pasha SF, Leighton JA, Das A, et al. Double-balloon enteroscopy and capsule endoscopy have comparable diagnostic yield in small-bowel disease: a meta-analysis. Clin Gastroenterol Hepatol 2008;6:671-676.

13. Matsumura T, Arai M, Saito K, et al. Predictive factor of re-bleeding after negative capsule endoscopy for obscure gastrointestinal bleeding: over 1-year follow-up study. Dig Endosc 2014;26:650-658.

14. Pérez-Cuadrado Robles E, Bebia Conesa P, Esteban Delgado P, et al. Emergency double-balloon enteroscopy combined with real-time viewing of capsule endoscopy: a feasible combined approach in acute overt-obscure gastrointestinal bleeding? Dig Endosc 2015;27:338-344. 